\title{
Clinico-Physiological and Molecular Characterization of Asthma-COPD Overlap Syndrome- A Hospital based Observational Study
}

\author{
Thiagu N ${ }^{1}$, C.V. Nandeshwara Reddy ${ }^{2}$, K.V. Giriraja ${ }^{3}$ \\ ${ }^{1}$ Post Graduate, Department of General Medicine, ${ }^{2}$ Assistant Professor, Department of Pulmonary Medicine, ${ }^{3}$ Professor, \\ Department of General Medicine, MVJ Medical College and Research Hospital, Bangalore, Karnataka, India
}

Corresponding author: C.V.Nandeshwara Reddy, Assistant Professor, Department of Pulmonary Medicine, MVJ Medical College and Research Hospital, Hoskote, Bangalore, 562114, India

DOI: http://dx.doi.org/10.21276/ijcmsr.2020.5.2.13

How to cite this article: Thiagu N, C.V. Nandeshwara Reddy, K.V. Giriraja. Clinico-physiological and molecular characterization of asthma-COPD Overlap syndrome- a hospital based observational study. International Journal of Contemporary Medicine Surgery and Radiology. 2020;5(2):B55-B59.

\section{A B S T R A C T}

Introduction: COPD and Asthma are high prevalent diseases with distinctly different genotype and phenotype. However, some patients present with mixture of both diseases which is recognized as Asthma - COPD Overlap Syndrome (ACOS). There is a need to explore biomarkers for ACOS to facilitate its precise diagnosis. Thus aim of the study was to study the characteristics and evaluate various diagnostic markers that can identify ACOS from COPD.

Materials and methods: 40 individuals who fulfilled the inclusion criteria were included as subjects in the study. A detailed history was taken followed by a clinical examination. Then the subjects performed the FeNO and Spirometry with bronchodilator response respectively. Following this, fasting venous blood sample was collected for performing the Absolute eosinophil count. The serum samples were assayed for periostin and Th1 and Th2 Interleukin panel.

Results: Serum Periostin and FeNO values are higher in ACOS than COPD only patients and is statistically significant. There is no statically significant difference among spirometry study, absolute eosinophil count, Th1 and Th2 Interleukin panel between two groups.

Conclusion: Study has found that serum periostin and FeNO are two biomarkers have fair diagnostic properties in identifying subjects with ACOS.

Keywords: Asthma, COPD, Asthma COPD Overlap Syndrome, Serum Periostin, FeNO

\section{INTRODUCTION}

Chronic Obstructive Pulmonary Disease (COPD) and Asthma are high prevalent diseases with significant health and economic burden to the society. COPD is characterized by persistent respiratory symptoms and airflow limitation that is due to airway and/or alveolar abnormalities usually caused by significant exposure to various particles or gases. There were about 384 million estimated cases of COPD in 2010. By 2030 the predicted cases annually will be over 4.5 million. World health organization predicts that by 2020 , COPD will become third leading cause of death (currently fourth) and fifth leading cause of disability (currently twelfth) worldwide. The greatest part on total COPD burden on healthcare is mainly due to the exacerbations. ${ }^{1,2}$

Asthma is a heterogenous disease, defined by the history of respiratory symptoms such as wheeze, shortness of breath, chest tightness and cough that vary over time and in intensity, together with variable expiratory airflow limitation. Asthma affects about 100-150 million people globally and causing over 1.8 lakhs death annually. In India, the prevalence of asthma varies from $2-6 \%$, with an estimated $15-20$ million asthmatics requiring special care. ${ }^{3}$

Asthma and COPD are traditionally considered as separate entities with different clinic-pathogenetic features. The symptoms and airflow limitation vary over time and intensity, they might sometimes resolve spontaneously or by medications in asthmatics, whereas in COPD patients they are found to have persistent airflow limitation. Immune response in Asthma, is type 2 mediated, inflammation is mainly eosinophilic and mediated by CD4+ T lymphocytes whereas for COPD there occurs neutrophilic inflammation driven by $\mathrm{CD} 8+\mathrm{T}$ lymphocytes.

Some patients present with mixture of both diseases which is recognized in recent years as Asthma - COPD Overlap Syndrome (ACOS). ACOS is characterized by persistent airflow limitations with some features usually associated with asthma and several features associated with COPD. ${ }^{1,3}$ Generally, ACOS subjects are younger, have more sputum, chest tightness, wheezing and night-time and day-time symptoms and significantly higher number of exacerbations, emergency department visits, hospital admissions, ICU admissions than patients with COPD-only or asthma-only. It is proposed that subjects with ACOS could have the 
combination of both eosinophilic mediated inflammations along with neutrophilic inflammation thereby having features of both asthma as well as COPD.

Firstly, treatment strategies for these conditions differ significantly. The principal treatment of choice for asthma is inhaled corticosteroids and so it is for ACOS. Inhaled steroids are recommended only for those COPD patients with severe-to-very severe airflow limitation and frequent exacerbations. Unjustified use of inhaled corticosteroids is known to increase the risk of pneumonia among COPD patients, inadvertent use of long acting beta adrenergic agonists without inhaled corticosteroids may be catastrophic in the patients with ACOS. secondly, patients presenting with ACOS have worse morbidity and mortality outcomes than those with COPD alone.

It is very essential to identify this kind of airway disorder to provide the apt treatment for the patients and thereby reducing the burden of morbidity and mortality caused by them. Hence the precise diagnosis of ACOS plays an important role not only to just categorize the patient but more than that to provide them with appropriate treatment and safeguard them from unwanted complications of a generalized treatment.

The various biomarkers being studied were like the sputum eosinophils, sputum neutrophil gelatinase associated lipocalin (NGAL), blood absolute eosinophil count, fractional concentration of exhaled nitric oxide(FeNO) and serum IgE. Exploiting this scenario, we proposed to study the characteristics of ACOS and evaluate various diagnostic markers that can identify ACOS as a distinctive phenotype from other obstructive airway diseases especially COPD.

\section{MATERIAL AND METHODS}

The present study was a prospective case control study conducted in department of medicine, MVJ Medical College and Research Hospital, Bangalore from July 2019 to February 2020. Consent was obtained from the patients participated in the study.

A total of forty patients with age of onset of disease above 40 years, diagnosis of ACOS and COPD according to the definition proposed by D.D. Sin et al. ${ }^{4}$ and Global initiative for obstructive lung disease (GOLD) guidelines definition ${ }^{2}$ respectively. Patients with Exacerbations of ACOS / COPD within two weeks prior to recruitment, active infections, chronic inflammatory disorders, malignancy, poorly controlled diabetes mellitus ( $\mathrm{HbA} 1 \mathrm{c}>8.5 \%$ within one month prior to recruitment) and decompensated cardiovascular disease, pregnancy are excluded from the study. Sampling done by convenient sampling method. The sample size was constituted by 20 subjects with ACOS and 20 subjects with COPD alone. The patients participated had detailed history taking followed by a clinical examination. The socio-demographic characteristics such as age, gender, socioeconomic status according to modified Prasad's scale, BMI, the place of dwelling such as urban or rural were all noted. Then the subjects performed the FeNO and Spirometry with bronchodilator response respectively. Following this, fasting venous blood sample about $5 \mathrm{ml}$ was collected from each of the subjects, after performing the
Absolute eosinophil count, the samples were centrifuged at 3000 RPM and serum was separated and stored at -80 degree Celsius. This stored samples were assayed on a later date, for serum Periostin and serum Th1 and Th2 Interleukin panel by ELISA method and bead based immunoassay respectively. The values are obtained in $\mathrm{pg} / \mathrm{ml}$. The analysis of these parameters was done to compare any significant difference between the ACOS and COPD arm.

\section{RESULTS}

In our study forty subjects participated, among them 38 (95\%) were males and $2(5 \%)$ were female. The age of the patients ranged from 40 to 72 years with a mean of $59.85 \pm 8.9$ years in the ACOS arm and $61.80 \pm 9.62$ years in the COPD arm. Males were dominant and constituted about $90 \%$ in ACOS and 100\% in COPD group. The BMI of subjects in ACOS group was found to be $20.76 \pm 2.7 \mathrm{~kg} / \mathrm{m} 2$ and 21.25 \pm 4.19 in the COPD group. Smoking was prevalent in most of the subjects included in the study, it was seen to be $90 \%$ in ACOS and 100\% in COPD group. Regarding the dwelling of patients which was thought might to have some impact on the disease status somehow was found to have urban dwelling of $70 \%$ in patients with ACOS and $65 \%$ in patients with COPD. The sociodemographic profile of the patients in ACOS and COPD alone group was almost comparable with not much significance.

On analysing the clinical characteristics of the patients in both the groups it was found that the duration of illness in the ACOS was $24.35 \pm 16.14$ years whereas in COPD group it was found to be $6.75 \pm 7.62$ years. That was statistically significant with a p value 0.0001 . Patients with more than one exacerbation in the last one year was almost similar in both arms with rate of $65 \%$. Among the various comorbidities like systemic hypertension, coronary artery disease, type 2 diabetes mellitus and others considered, the subjects had Charlson's comorbidity index of $2.7 \pm 1.03$ in ACOS and in the COPD group was $3.2 \pm 1.32$. Among the various clinical characteristics considered the duration of illness in the patients with ACOS was found to be significantly longer than those with COPD alone.

The physiological characteristics when analysed based on the spirometry values it was found that none of the spirometry parameters were different between the groups and it was comparable.(Table.1)

Among the various inflammatory characteristics studied it was found that the ACOS subjects had significantly higher FeNO and higher serum periostin levels as compared to the subjects with COPD alone. The serum periostin levels obtained by the ELISA method was found to be $423.2 \pm$ $609.1 \mathrm{ng} / \mathrm{mL}$ whereas in COPD it was only $101.6 \pm 119.2$ $\mathrm{ng} / \mathrm{mL}$. This was found to be statistically significant with a $\mathrm{P}$ value of 0.026.(Table.2)

The association between serum Periostin and ACOS was independent of age and Body Mass Index (BMI) with an adjusted Odds ratio 1.006 with 95\% confidence interval 1.001 to 1.010 , with $\mathrm{p}$ value 0.026 . On assessing the diagnostic performance under ROC curve, Periostin showed a fair discriminative property with area under ROC 0.765 with sensitivity and specificity of $75 \%$ with a cutoff of 110 


\begin{tabular}{|l|c|c|c|}
\hline Variables & ACO S(n=20) & COPD $(\mathbf{n}=\mathbf{2 0})$ & p Value \\
\hline Pre FVC \% predicted & $68.57 \pm 15.9$ & $67.17 \pm 21.6$ & 0.817 \\
\hline PreFEV1\% Predicted & $45.6 \pm 14.6$ & $52.05 \pm 19.7$ & 0.249 \\
\hline Pre FEV1 / FVC & $43.75 \pm 7.83$ & $44.29 \pm 18.79$ & 0.905 \\
\hline PostFVC\% Predicted & $81.9 \pm 19.0$ & $74.78 \pm 24.1$ & 0.306 \\
\hline PostFEV1\%predicted & $57.55 \pm 17.8$ & $61.25 \pm 22.5$ & 0.567 \\
\hline Post FEV1/FVC & $56.48 \pm 8.8$ & $50.16 \pm 18.9$ & 0.884 \\
\hline PreMEF25-75\% predicted & $17.57 \pm 7.71$ & $23.84 \pm 15.8$ & 0.119 \\
\hline PostMEF25-75\% Predicted & $22.87 \pm 30.4$ & $10.27 \pm 20.92$ & 0.158 \\
\hline \multicolumn{2}{|r|}{} \\
\hline
\end{tabular}

\begin{tabular}{|l|c|c|c|}
\hline Variables & ACOS (n=20) & COPD (n=20) & p Value \\
\hline Absolute Eosinophil Count (Blood) & $535 \pm 598.5$ & $421.5 \pm 536.7$ & 0.532 \\
\hline Fractional Exhaled Nitric Oxide (FeNO in ppb) & $23.45 \pm 18.53$ & $13.60 \pm 6.168$ & 0.030 \\
\hline Serum.Periostin (ng/mL) & $423.2 \pm 609.1$ & $101.6 \pm 119.2$ & 0.026 \\
\hline \multicolumn{2}{|r|}{ Table-2: Inflammatory Characteristics of the Study Population } \\
\hline
\end{tabular}

\begin{tabular}{|l|c|c|c|}
\hline Variables & ACOS $\mathbf{( n = 2 0 )}$ & COPD $(\mathbf{n = 2 0})$ & p Value \\
\hline Interleukin-2 $(\mathrm{pg} / \mathrm{mL})$ & $5.27 \pm 3.96$ & $4.13 \pm 1.82$ & 0.216 \\
\hline Interleukin-4 $(\mathrm{pg} / \mathrm{mL})$ & $11.44 \pm 10.78$ & $7.02 \pm 5.12$ & 0.114 \\
\hline Interleukin-5 $(\mathrm{pg} / \mathrm{mL})$ & $5.08 \pm 3.63$ & $4.14 \pm 2.38$ & 0.268 \\
\hline Interleukin-6 $(\mathrm{pg} / \mathrm{mL})$ & $16.7 \pm 19.77$ & $7.18 \pm 6.3$ & 0.069 \\
\hline Interleukin-10 (pg/mL) & $1.43 \pm 0.68$ & $1.4 \pm 0.36$ & 0.832 \\
\hline Interleukin-13 (pg/mL) & $3.17 \pm 2.0$ & $2.57 \pm 0.87$ & 0.198 \\
\hline Interferon - gamma $(\mathrm{pg} / \mathrm{mL}) \quad 17.9 \pm 25.7$ & $11.0 \pm 7.55$ & 0.260 \\
\hline \multicolumn{2}{|r|}{ Tumor necrosis factor - alpha $(\mathrm{pg} / \mathrm{mL})$} & $5.99 \pm 2.91$ & 0.320 \\
\hline \multicolumn{2}{|r|}{ Table-3: Interleukin panel Characteristics of the Study Population } \\
\hline
\end{tabular}

A6.IL-2

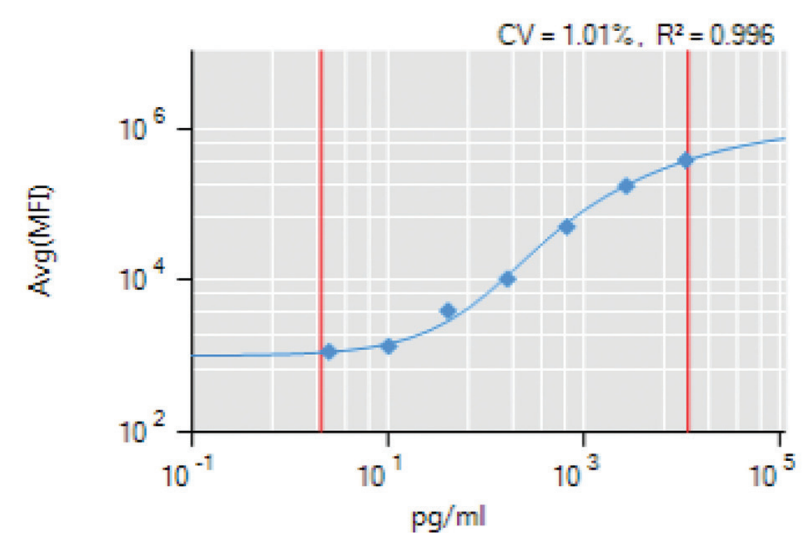

Figure-1:

$\mathrm{ng} / \mathrm{mL}$ and above. (fig.1)

In ACOS patients FeNO was $23.45 \pm 18.53 \mathrm{ppb}$ but in COPD patients it was only $13.60 \pm 6.168 \mathrm{ppb}$, the difference was statistically significant with p value 0.030 (Table 2). This association between FeNO and ACOS was independent of age and BMI, with an adjusted Odds ratio of 1.095 with $95 \%$ confidence interval 1.009 to 1.187 , with $\mathrm{p}$ value 0.030 . The diagnostic performance analysis showed FeNO with a fair discriminative property with area under ROC 0.735 with a sensitivity and specificity of $70 \%$ at a cut off of $20 \mathrm{ppb}$ and above. (Fig.2)

Absolute eosinophil count in the peripheral blood was
A7 $:$ IL -6

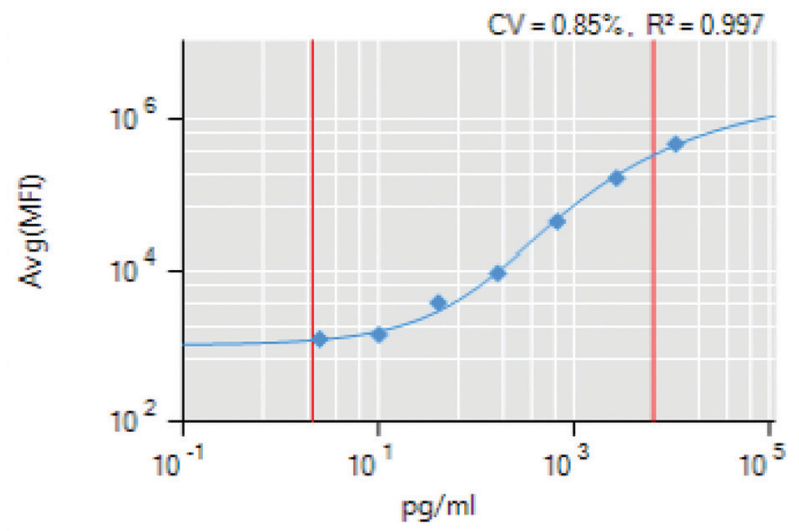

Figure-2:

not significantly different between subjects with COPD and ACOS. The extensive analysis of the interleukin panel involving the Th1 and Th2 cytokines revealed no difference between the groups.(Table.3)

\section{DISCUSSION}

As of now the disease entity of ACOS itself is considered as a new phenotype with features constituting those of asthma and COPD. This phenotype at present is on an evolving phase in its diagnostic and treatment aspects. There are no validated diagnostic criteria for ACOS. Moreover, there is a paucity in the biomarker availability for ACOS diagnosis, 
an appropriate biomarker is needed since they can ease the diagnosis in the busy outpatient settings.

Most studies have not included the subset of population with features of both asthma and COPD as they were conducted to analyse either asthma or COPD alone. We do not have a better insight on the sociodemographic profile and clinical characteristics of these patients.

In our study, it was found that the physiological characteristics among those with ACOS and COPD was comparable there was not much significant difference between them. The duration of illness was found to be higher in the subjects with ACOS than those with COPD alone. It was found to be statistically significant one.

In our study, serum Periostin in ACOS was found to be $423.2 \pm 609.1 \mathrm{ng} / \mathrm{mL}$ whereas in COPD it was only $101.6 \pm$ $119.2 \mathrm{ng} / \mathrm{mL}$, a statistically significant difference observed. The adjusted Odds ratio 1.006 with $95 \%$ confidence interval 1.001 to 1.010 , with $\mathrm{p}$ value 0.006 . Periostin showed a sensitivity and specificity of $75 \%$ with a cut off of $110 \mathrm{ng} /$ $\mathrm{mL}$. In the patients with ACOS, FeNO was $23.45 \pm 18.53$ ppb but in COPD patients it was only $13.60 \pm 6.168$ ppb, a statistically significant difference. The adjusted Odds ratio of 1.095 with $95 \%$ confidence interval 1.009 to 1.187 , with $\mathrm{p}$ value 0.030 . FeNO was found to have a sensitivity and specificity of $70 \%$ at a cut off of $20 \mathrm{ppb}$ and above. Absolute eosinophil count considered as a marker for eosinophilic inflammation was found to be comparable between the two groups. Guiquan Jia, MD et al in the BOBCAT study in the patients with asthma described the correlation of serum Periostin with airway eosinophilia and as a biomarker of airway eosinophilia. The levels of serum Periostin greater than $25 \mathrm{ng} / \mathrm{mL}$ were seen to have $85 \%$ probability of a composite airway eosinophil score of 1 or 2 . They found that on using $25 \mathrm{ng} / \mathrm{mL}$ serum periostin as an arbitrary cut off, eosinophillow and eosinophil-high subjects in BOBCAT are effectively differentiated, with a positive predictive value of $93 \% .^{5}$

The cytokines involved in inflammation were extensively studied using the Th1 and Th2 interleukin panel. None were found to be significantly different between the groups, thus needs more evaluation. In tropical countries like India where there are many causes like parasitic infestations are of considerable prevalence, relying on the markers like peripheral eosinophilia, serum $\operatorname{IgE}$ are not relevant and nonspecific for validating the diagnosis. Probably, for same reason, our study found no difference in peripheral eosinophilia between ACOS and COPD.

Also using sputum eosinophilia which has been extensively studied as a marker of eosinophilic airway inflammation in routine practice is a difficult one as it requires expertise from pathology laboratory.

Viswanathan Emprm et al, in their study with 101 subjects described the serum periostin as more sensitive in detecting the airflow limitation than other parameters like absolute eosinophils, sputum eosinophil counts. It was found that serum periostin had sensitivity and specificity of $97.18 \%$ and $86.67 \%$ with a diagnostic accuracy of $94.06 \%$ respectively. ${ }^{6}$

The studies like those mentioned above in the literature are found to have serum periostin as a biomarker with a probable cut off value which is less than that achieved in our study.
Moreover, the studies were done on patients with asthma and we could not find studies in the literature which have been performed in the patients with COPD or those with ACOS. Hence these values cannot be accepted as such when it comes to the patients with ACOS who are having the features of both asthma and COPD.

Tadahiro Goto, et al, in their work, FeNO levels in asthmaCOPD overlap syndrome, a cross-sectional analysis found that the levels of FeNO are higher in patients with $\mathrm{ACO}$ with mean of $21.2 \mathrm{ppb}$ than in those with COPD alone. Also, they found that FeNO level was significantly higher in non-current smokers with ACOS than non-smokers with COPD alone. ${ }^{7}$

Yuanyuan Guo, MD, et al, in their case control study involving 106 patients with 53 in ACOS and 53 in COPD group showed FeNO levels in patients with ACOS (37[24.553.0] $\mathrm{ppb}$ ) was significantly higher than those of patients with COPD alone (20 [15.5-24.5] ppb). The area under the receiver operating curve of FeNO for the diagnosis of ACOS phenotype was 0.815 , the sensitivity and specificity to be highest when the cut off value was $25.50 \mathrm{ppb}^{8}$ Our study showed a very similar diagnostic performance of $\mathrm{FeNO}$ in discriminating ACOS from COPD alone.

FeNO is considered as a marker of Th2 mediated, eosinophilic inflammation in the patients with asthma. There are many studies of FeNO in asthma. The literature search showed studies in which FeNO was used as marker of eosinophilic inflammation, to identify the same in patients with COPD. Thereby FeNO can be a diagnostic marker to identify the phenotype of ACOS. The findings of our study are also consistent with those found in literature.

Shironjit K Saha, et al in their study proposed that IL-13 over expression is seen in patients with severe asthma with increased sputum IL-13 concentration and IL-13 ${ }^{+}$cells in the bronchial submucosa and airway smooth muscle bundle. Asthma control and the intensity of eosinophilic inflammation were found to have an association with IL-13 expression. It can be considered to use periostin levels as a biomarker for anti-IL-13 therapy since it has been postulated that IL 13 interleukin has been found to be associated with synthesis of periostin. In our study though we had a positive correlation for periostin in patients with ACOS, IL $13 \mathrm{did}$ not have much significant difference on comparison between ACOS and COPD only. Thus, serum periostin and FeNO can be considered as a novel biomarker in the diagnosis of ACOS. ${ }^{9}$

The literature search showed not much studies which have analysed the various interleukins in patients with ACOS. These interleukins could possibly be more pronounced during exacerbation of ACOS. However, our study was designed to recruit subjects in remission state and probably thus we could not elicit any difference in Th2 interleukin response in ACOS.

Chronic airflow limitation can be confirmed by spirometry, the ability is limited to distinguish between asthma with fixed airflow obstruction, COPD and ACOS.

Satoru Yanagisawa, et al, in their work on ACOS proposed that in asthmatics, airway reversibility is common, but in those who have undergone airway remodelling, absence of 
reversibility does not necessarily rule out the presence of asthma. Even in some COPD subjects a post bronchodilator response of FEV1 $\geq 400 \mathrm{~mL}$ or $15 \%$ from the baseline in patients with COPD strongly suggests the co-existence of asthma. ${ }^{10}$

In our study it was found that there was no positive correlation of the physiological characteristics studied using spirometry. Though there are many studies that show a positive post bronchodilator response which is considered as criteria for diagnosis of ACOS, our study did not have so.

\section{Limitations}

A small sample size due time bound nature of our study and financial constraints, very few female subjects were recruited since smoking was less prevalent in our society and exposure to biomass fuel smoke was unreliable and could not be quantified or equated to smoking intensity criteria as indicated in ERS Consensus statement on definition of ACOS

\section{CONCLUSION}

Our study has found that serum periostin and FeNO are two biomarkers that have fair diagnostic properties especially when combined, in identifying subjects with ACOS. These two diagnostic markers appear to be specific, reliable and less expensive and thus relevant for routine use in clinical practice. We recommend studies in larger samples, that could bring out diagnostic models with both clinical variables and biomarkers. Such diagnostic models, we believe could ultimately revolutionize our current way of defining and managing ACOS.

\section{REFERENCES}

1. GOLD-2018-v6.0-FINAL-revised-20-Nov_WMS. pdf [Internet]. [cited 2018 Aug 11]. Available from: https://goldcopd.org/wp-content/uploads/2017/11/ GOLD-2018-v6.0-FINAL-revised-20-Nov_WMS. pdf.

2. GOLD 2017 Global Strategy for the Diagnosis, Management and Prevention of COPD [Internet]. Global Initiative for Chronic Obstructive Lung Disease - GOLD. [cited 2018 Oct 15]. Available from: https:// goldcopd.org/gold-2017-global-strategy-diagnosismanagement-prevention-copd.

3. (*NEW) 2018 GINA Report: Global Strategy for Asthma Management and Prevention [Internet]. Global Initiative for Asthma - GINA. [cited 2018 Aug 11].

4. Ding B, Enstone A. Asthma and chronic obstructive pulmonary disease overlap syndrome (ACOS): structured literature review and physician insights. Expert Rev Respir Med. 2016;10(3):363-71.

5. Jia G, Erickson RW, Choy DF, Mosesova S, Wu LC, Solberg OD, et al. Periostin is a systemic biomarker of eosinophilic airway inflammation in asthmatic patients. J Allergy Clin Immunol. 2012;130(3):647-654.e10.

6. Emprm V, Rajanandh M, Nageswari A. Periostin - A Novel Systemic Biomarker for Eosinophilic Airway Inflammation: A Case Control Study. J Clin Diagn Res JCDR. 2016 Feb;10(2):OC01-4.

7. Goto T, Camargo CA, Hasegawa K. Fractional exhaled nitric oxide levels in asthma-COPD overlap syndrome: analysis of the National Health and Nutrition Examination Survey, 2007-2012. Int J Chron Obstruct Pulmon Dis. 2016;11(2):2149-55.

8. Guo Y, Hong C, Liu Y, Chen H, Huang X, Hong M. Diagnostic value of fractional exhaled nitric oxide for asthma-chronic obstructive pulmonary disease overlap syndrome. Medicine (Baltimore). 2018;97(23):e10857.

9. Saha SK, Berry MA, Parker D, Siddiqui S, Morgan A, May R, et al. Increased sputum and bronchial biopsy IL13 expression in severe asthma. J Allergy Clin Immunol. 2008;121(3):685-91.

10. Yanagisawa S, Ichinose M. Definition and diagnosis of asthma-COPD overlap (ACO). Allergol Int. 2018;67(2):172-8.

\section{Source of Support: Nil; Conflict of Interest: None}

Submitted: 17-03-2020; Accepted: 06-05-2020; Published online: 02-06-2020 